\title{
Monte Carlo Simulations of Ferromagnetic Nanocomposites
}

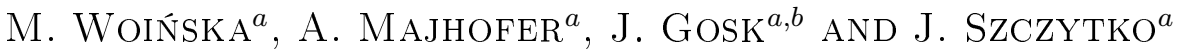 \\ ${ }^{a}$ Institute of Experimental Physics, Faculty of Physics, University of Warsaw, Hoża 69, 00-681 Warsaw, Poland \\ ${ }^{b}$ Faculty of Physics, Warsaw University of Technology, Koszykowa 75, 00-662 Warsaw, Poland
}

We present results of Monte Carlo simulations of a model system of Co nanoparticles in an external magnetic field. In our simulations we take into account the interaction with the external magnetic field, the energy of crystallographic anisotropy and the dipole-dipole interactions between single domain nanoparticles. We apply the standard Metropolis algorithm to a cluster of 27 randomly distributed superparamagnetic Co nanoparticles. We investigate the dependence of blocking temperature $T_{\mathrm{B}}$ on particle and cluster size.

PACS: $75.40 . \mathrm{Mg}, 75.50 . \mathrm{Tt}, 75.75 .-\mathrm{c}$

\section{Introduction}

There are several strategies to combine ferromagnetism and semiconducting properties of matter. One is to create ferromagnetic semiconductors - typically of III-V groups: GaAs:Mn or GaN:Mn and GaN:Fe. Much effort has been directed to develop growth techniques of these crystals with the concentration of magnetic ions high enough to approach room temperature ferromagnetism. However, these attempts usually result in the formation of ferromagnetic precipitations. It has been also reported that thermal annealing of $\mathrm{Ga}_{1-x} \mathrm{Mn}_{x}$ As layers leads to formation of MnAs dots, as well as that in metal oxide vapor phase epitaxy (MOVPE)-grown ( $\mathrm{Ga}, \mathrm{Fe}) \mathrm{N}$ ferromagnetic $\mathrm{FeN}_{x}$ nanocrystals aggregate by precipitations [1-4]. Such system is often called nanocomposite. Therefore the other strategy to have both: ferromagnetic and semiconducting matter, is to use these nanocomposites, which can also be promising candidates for information storage and spin electronics applications.

The problem which occurs is that such complex systems need theoretical modeling different than just simple virtual crystal approximation. For instance when modeling ferromagnetic (or superparamagnetic) properties it is necessary to include crystalline anisotropy of single-crystal domains within each ferromagnetic nanoparticle as well as its interaction with external magnetic field and other nanoparticles randomly distributed in space.

\section{Monte Carlo simulations}

To better understand the behavior of a system of ferromagnetic precipitations in a non-magnetic host we choose a system of Co-based nanoparticles embedded in diamagnetic host. Such systems with well-known particle sizes can be produced in a controlled chemical synthesis. This property allows for comparisons of calculations with experimental results. In our simulations we take a cluster of 27 randomly distributed superparamagnetic Co nanoparticles. The model Hamiltonian includes: crystallographic anisotropy $K=-2.7 \times 10^{6} \mathrm{erg} / \mathrm{cm}^{3}$ (corresponding to fcc Co single crystals [6]), coupling to the homogeneous external magnetic field and the magnetic dipole-dipole interactions between nanoparticles. Since the temperature range we are interested in lies far below the Curie-temperature of Co, each particle is represented as a magnetic dipole moment equal to the sum of moments of Co atoms it is composed of, located in the center of a particle (thus we assume single-domain nanoparticles [7]). We assume particles to be spherical, equal in size and we consider particles of diameter $d$ from $3 \mathrm{~nm}$ to $13 \mathrm{~nm}$. Directions of crystallographic axes of particles are chosen randomly and are fixed during calculations. By simply rescaling the distances between the particles we can easily simulate (and compare) systems of different density of superparamagnetic nanoparticles. The scaling factor (s.f.) is equal to the factor by which distances between particles are multiplied with respect to the smallest cluster $($ s.f. $=1$ ) in which each particle touches at least its one neighbor. Thus, in our model, we can investigate how the result of competition between the anisotropy energy and magnetic dipole-dipole interactions depends on the size of particles and their density. In our simulations we use the standard Metropolis algorithm [8, 9] and calculate zero-field-cooled (ZFC) and field-cooled (FC) magnetization curves of the system in the temperature range of $2.0-400.0 \mathrm{~K}$. We take the same number of Monte Carlo steps (MC steps - in one MC step magnetic moments of all the particles are rotated) to thermalize the system after changing the temperature and to calculate thermal averages. The $\mathrm{ZFC}$ and $\mathrm{FC}$ curves split below certain blocking temperature $T_{\mathrm{B}}$. The latter depends on the external field, the rate of temperature changes, sizes of superparamagnetic particles and on the distances between nanoparticles. To investigate the 
dependence of the blocking temperature $T_{\mathrm{B}}$ on the rate of changes of the external field we repeat our ZFC-FC calculations for different numbers of MC steps the system evolves at constant temperature. To simulate an infinite system of superparamagnetic nanoparticles we periodically repeat the cluster and use Ewald summation procedure to properly account for long-range dipole-dipole interactions [10-12].

\section{Results and discussion}

In Fig. 1 we present ZFC-FC magnetization curves calculated for a system of 27 particles of diameter $d=11 \mathrm{~nm}$ in the external field $B=0.01 \mathrm{~T}$, for three scaling factors s.f. $=1,2,8$ and three different numbers of MC steps: $1000,5000,50000$. The results displayed in Fig. 1a correspond to a single cluster and are averaged over 20 different configurations of particles within the cluster (i.e. different random distributions of nanoparticles). These results can be compared with the preliminary results (only one configuration) obtained for an "infinite system" shown in Fig. 1b. It is easily seen that the ZFC-FC splitting considerably grows with increasing distances between particles and decreases with increasing number of MC steps. This splitting is larger in the case of infinite system.

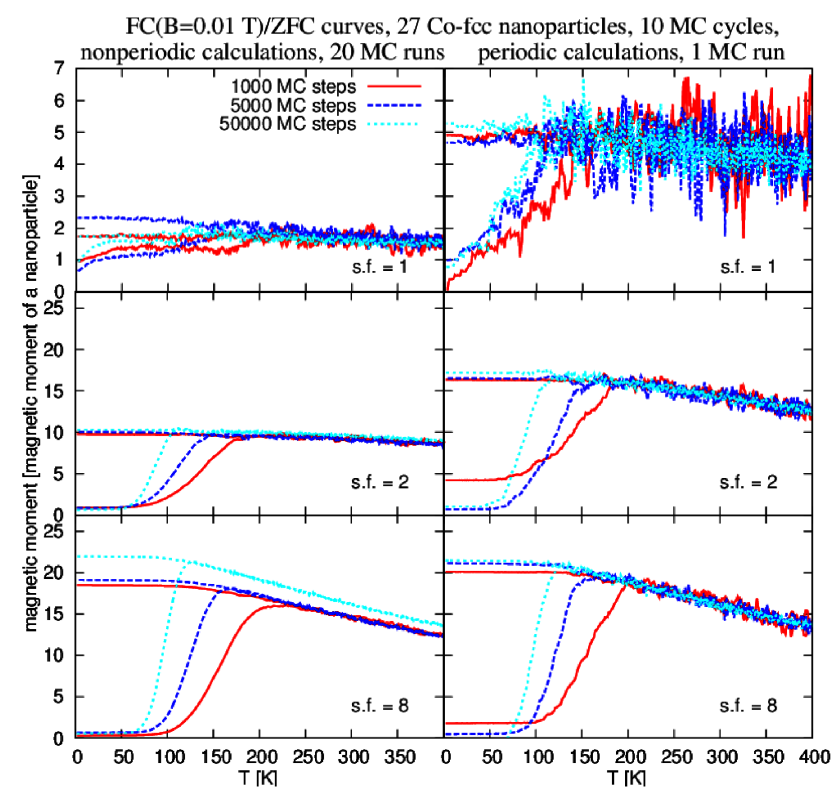

Fig. 1. (a) Results of Monte Carlo simulations of a single cluster averaged over 20 different configurations of 27 nanoparticles within the cluster. (b) Results obtained for an "infinite system" according to Ewald summation procedure.

The splitting in $\mathrm{ZFC}-\mathrm{FC}$ measurements is due to the energy barriers present in the system of magnetic nanoparticles - at low temperature the magnetization of particles is blocked at certain configuration which is not a global energetic minimum of the whole system. The system can find its way to the global minimum either by increasing the temperature and allowing thermal fluctuations to overcome energetic barriers or by waiting for sufficient time (which is in many cases comparable to the age of Universe). In Monte Carlo modeling the number of MC steps is proportional to the duration time of the experiment. Therefore more MC steps means longer waiting time. To summarize our observations, the estimated values of $T_{\mathrm{B}}$ as functions of the number of $\mathrm{MC}$ steps are displayed in Fig. 2. As expected, $T_{\mathrm{B}}$ falls when temperature changes are slower - i.e. the number of $\mathrm{MC}$ steps is larger.

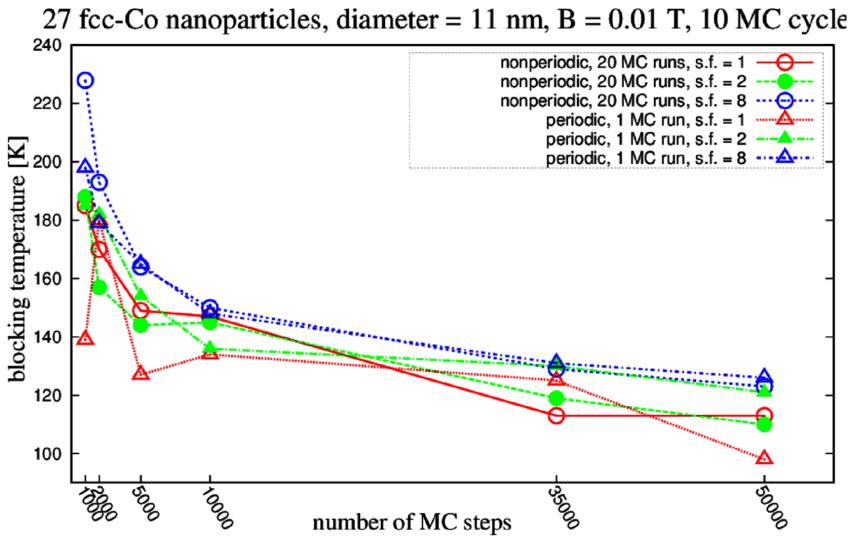

Fig. 2. The estimated values of $T_{\mathrm{B}}$ as functions of the number of MC steps.

Magnetic properties of nanocomposites strongly depend on two length scales: sizes of particles and their average distances. Our model naturally allows for investigations of the interplay between them as we can easily change particle diameters and put them closer or further apart, when varying values of the scaling factor s.f. In Fig. 3 we display $T_{\mathrm{B}}$ as a function of particle diameter $d$ for a range of values from $3 \mathrm{~nm}$ to $13 \mathrm{~nm}$ for s.f. $=1,2$ and 8 and the number of MC steps equal to 10000 . In this case the results for finite and infinite system are much the same.

As expected, and well known from experiments on nanocomposites, $T_{\mathrm{B}}$ grows with increasing $d$. When particles are small $(d \leq 11 \mathrm{~nm}), T_{\mathrm{B}}$ does not change with increasing s.f., but for $d>11 \mathrm{~nm}$ we observe its rapid growth when s.f. is changed from 1 to 8 . This is an interesting manifestation of the interplay between the anisotropy energy and the long-range dipole-dipole interactions. When particles are small, the dipole-dipole interactions are weaker than the anisotropy barriers even for the closest nanoparticles and the system behaves in a similar way as a system of non-interacting superparamagnetic particles. For large particles dipole-dipole interactions dominate at short interparticle distances while anisotropy energy prevails for larger distances. We thus conclude that there exists a "critical" particle size above which blocking temperature of a system strongly depends on characteristic interparticle distances. This might be 


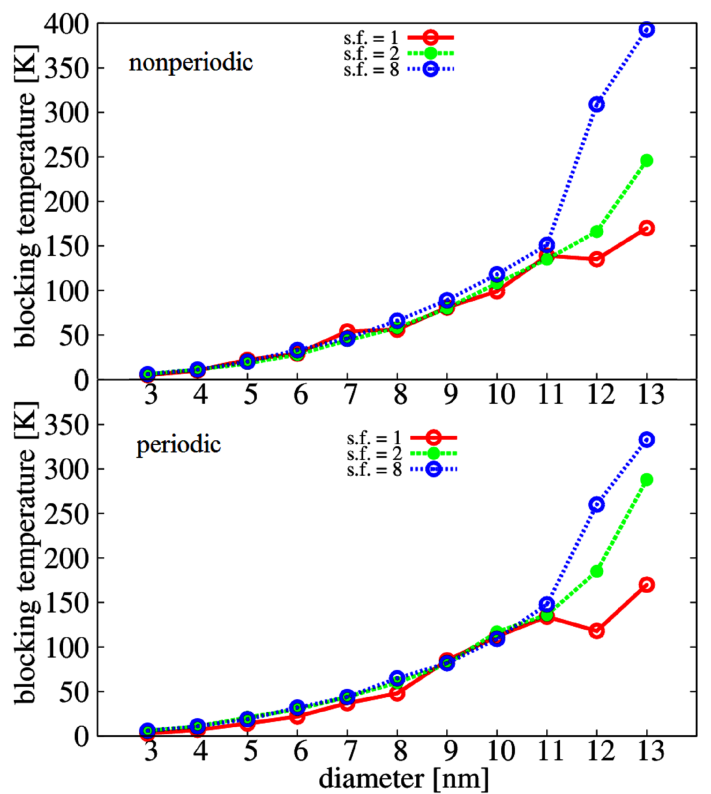

Fig. 3. The estimated values of $T_{\mathrm{B}}$ as functions of the diameter of nanoparticle for nonperiodic (upper figure, 20 runs) and periodic (lower figure, 1 run) calculations. External magnetic field $B=0.01 \mathrm{~T}, 10000 \mathrm{MC}$ steps.

of interest for prospective technological applications of magnetic nanocomposites. For a system of single-domain cobalt particles considered here, such critical diameter is equal to $d=11 \mathrm{~nm}$, less than estimated maximum size of one-domain Co nanoparticle (diameter of about $20 \mathrm{~nm}$ [6]). Preliminary results of simulations for an infinite medium show the same behavior and thus corroborate our conclusion concerning the existence of some critical size of nanoparticles above which $T_{\mathrm{B}}$ strongly depends on interparticle distances.

\section{Summary}

In this short report we discuss the results of Monte Carlo simulations of a system of monodisperse superparamagnetic, spherical, single-domain nanoparticles embedded in a magnetically inert host. We calculate ZFC and FC magnetization curves to investigate blocking temperature as a function of particle diameter and interparticle distance. Our main conclusion is that there exists critical size of the particles above which blocking temperature strongly depends on interparticle distances. This result might be of interest for technological applications of nanocomposites containing superparamagnetic particles, and as far as we know it has not yet been discussed in the literature.

\section{References}

[1] T. Dietl, H. Ohno, F. Matsukura, Phys. Rev. B 63, 195205 (2001).

[2] T. Jungwirth, K.Y. Wang, J. Mašek, K.W. Edmonds, J. König, J. Sinova, M. Polini, N.A. Goncharuk, A.H. MacDonald, M. Sawicki, R.P. Campion, L.X. Zhao, C.T. Foxon, B.L. Gallagher, Phys. Rev. B 72, 165204 (2005).

[3] F. Matsukura, M. Sawicki, T. Dietl, D. Chiba, H. Ohno, Physica E 21, 1032 (2004).

[4] A. Bonanni, M. Kiecana, C. Simbrunner, Tian Li, M. Sawicki, M. Wegscheider, M. Quast, H. Przybylińska, A. Navarro-Quezada, R. Jakieła, A. Wolos, W. Jantsch, T. Dietl, Phys. Rev. B 75, 125210 (2007).

[5] A. Bonanni, A. Navarro-Quezada, Tian Li, M. Wegscheider, Z. Matej, V. Holy, R.T. Lechner, G. Bauer, M. Rovezzi, F. D'Acapito, M. Kiecana, M. Sawicki, T. Dietl, Phys. Rev. Lett. 101, 135502 (2008).

[6] W. Sucksmith, J.E. Thompson, Proc. R. Soc. Lond. A 225, 362 (1954).

[7] W. Gong, H. Li, Zh. Zha, J. Chen, J. Appl. Phys. 69, 5119 (1991).

[8] N. Metropolis, A.W. Rosenbluth, M.N. Rosenbluth, A.H. Teller, E. Teller, J. Chem. Phys. 21, 1087 (1953).

[9] D.W. Heermann, K. Binder, Monte Carlo Simulation in Statistical Physics, Springer-Verlag, Berlin 1992.

[10] P. Ewald, Ann. Phys. 369, 253 (1921).

[11] W. Cai, H. Lee, Ewald Summation for Coulomb Interactions in a Periodic Supercell, Department of Mechanical Engineering, Stanford University, CA 94305-4040, 2009.

[12] D.J. Tildesley, M.P. Allen, Computer Simulation of Liquids, Oxford University Press, New York 1987. 